\section{Comparison of clinical outcome with different treatment regimens in acute adenoviral keratoconjunctivitis}

L Asena', E Şıngar Özdemir², A Burcu', E Ercan', M Çolak ${ }^{3}$ and DD Altınörs ${ }^{1}$

\begin{abstract}
Purpose To compare the clinical outcome with different treatment regimens in Acute Adenoviral Keratoconjunctivitis (AAK).

Methods The records of 110 patients diagnosed as AAK in two tertiary eye care centers were evaluated retrospectively. The treatment regimen, follow-up duration, time until improvement of the symptoms, visual acuity, clinical findings, Schirmer's test and the Ocular Surface Disease Index (OSDI) score at the first day, first week and third week were recorded. The Kruskal-Wallis Test and Chi-square test were used for comparison of quantitative and categorical variables, respectively.

Results Forty six patients were receiving topical corticosteroids (Group 1), 32 topical 2\% Cyclosporin A (CsA) (Group 2) and 32 only artificial tears (Group 3). Mean time until resolution of the symptoms was lowest in Group 1 (9.5 \pm 4.9 days) and highest in Group 3 (13.3 \pm 4.2 days)( $p$ : 0.001$)$. OSDI scores at the first and third weeks were highest in Group $3(52.4 \pm 16.4$ and $32.3 \pm 13.0)$ and lowest in Group $1(41.0 \pm 21.3$ and $23.9 \pm 13.2)(p: 0.01$ and $p$ : 0.009). At day 21 , percentage of the patients with subepthelial infiltrates was the highest in Group $3(63.4 \%)$ and lowest in Group 1 (42.5\%).

Conclusions The symptoms were less severe and had a shorter duration with topical corticosteroids and CsA when compared with palliative therapy. Topical $2 \%$ CsA may inhibit development of corneal subepithelial infiltrates when used in the acute phase of infection, similar to corticosteroids.
\end{abstract}

Eye (2017) 31, 781-787; doi:10.1038/eye.2017.4; published online 3 February 2017

\section{Introduction}

Acute adenoviral keratoconjunctivitis (AAK), which was first described by Fuchs ${ }^{1}$ in 1889 is the most common external ocular viral infection worldwide. It may present epidemically or sporadically and is highly contagious. The natural course of the acute phase of AAK has a wide spectrum of duration and intensity of local symptoms. After an incubation period of 2-14 days, typical symptoms including conjunctival hyperaemia and chemosis, swelling of the conjunctival plica, and intense tearing usually begin in one eye. ${ }^{2}$ Different from other forms of viral conjunctivitis, corneal

involvement and impaired vision are important complications of AAK. During the course of the infection, $\sim 10$ days after onset of the symptoms, corneal subepithelial opacities frequently develop.

The American Academy of Ophthalmology Preferred Practice Pattern proposes symptomatic treatment in the form of artificial tears, topical antihistamines, or cold compresses to mitigate symptoms and states that there is inadequate evidence to support the use of available antiviral agents for treating adenoviral conjunctivitis. ${ }^{3}$

The use of topical steroids is recommended only to reduce scarring in severe cases of adenoviral keratoconjunctivitis with marked chemosis, lid swelling, epithelial sloughing, or membranous conjunctivitis. However, short term topical steroids are still used with an aim to decrease patient discomfort. ${ }^{4}$

Cyclosporine A, which is a well known immunosuppressive agent, has been used for the prevention of transplant rejections for a long time. ${ }^{5}$ Topical Cyclosporine A (CsA) has been used effectively in the treatment of many ocular surface diseases such as keratoconjunctivitis
${ }^{1}$ Department of Ophthalmology, Baskent University Faculty of Medicine, Ankara, Turkey

${ }^{2}$ Department of Ophthalmology, Ankara Education and Research Hospital, Ankara, Turkey

${ }^{3}$ Department of Biostatistics, Baskent University, Health Sciences Faculty, Ankara, Turkey

Correspondence:

L Asena, Baskent University Faculty of Medicine, Fevzi Çakmak Caddesi, Bahçelievler, Ankara 06490, Turkey

Tel: +90 31221503 49; Fax: +90 3122237333 . E-mail: leylaerk@yahoo.com

${ }^{4}$ Present Address: Kafkas University Faculty of Medicine, Department of Ophthalmology,

Kars, Turkey

Received: 14 January 2016 Accepted in revised form: 28 October 2016; Published online: 3 February 2017 
sicca, ${ }^{6}$ atopic and vernal keratoconjunctivitis, ${ }^{7}$ ulcerative keratitis, ${ }^{8}$ and Thygeson's punctate keratitis. ${ }^{9}$ It has also been used in varying concentrations between 0.5 and $2 \%$ for eradicating the symptoms and minimising the recurrences of corneal subepithelial infiltrates in acute adenoviral keratoconjunctivitis. ${ }^{10-13}$ Virustatic agents such as trifluridine, vidarabine, ganciclovir, and cidofovir have been found to be only mildly effective or entirely ineffective against adenoviral diseases, whether in vitro, in vivo, or in human clinical trials. ${ }^{14-16}$ Povidone-iodine is a non-causally-directed treatment option for AAK with a virucidal effect on adenoviruses. It was reported that povidone iodine reduced viral load and the severity of symptoms in vitro and in vivo studies for the treatment of epidemic keratoconjunctivitis. ${ }^{17,18}$ However, there are no controlled trials supporting the use of this non-specific treatment option.

There is still need for a safe and effective treatment modality to reduce patient discomfort, morbidity, absence time from school or work and to prevent developement of vision altering complications such as corneal subepithelial infiltrates. Although there are reports that show the efficacy of topical CsA (1 and 2\%) in decreasing the incidence of corneal opacities and in the therapy of active subepithelial infiltrates during the chronic phase, ${ }^{11-13,19,20}$ there are no studies reported to compare the safety and efficacy of topical Cyclosporine A with topical corticosteroids and palliative treatment in acute adenoviral keratoconjunctivitis. Therefore, we aimed to compare the clinical outcome with different treatment regimens including topical corticosteroids, Cyclosporin $\mathrm{A}$ and exclusively palliative therapy, in a large group of patients who admitted to two tertiary eye care centers.

\section{Patients and methods}

The study was approved by the Institutional Review Board of Baskent University Faculty of Medicine and adhered to the tenets of the Declaration of Helsinki. The records of the patients diagnosed as AAK in two tertiary eye care centers between April 2014 and September 2015 were evaluated retrospectively. The treatment regimen, follow-up duration, visual acuity, intraocular pressure, clinical findings including conjunctival injection, chemosis and subepithelial infiltrates, Schirmer's test results and the Ocular Surface Disease Index (OSDI) score at the first day, first week and third week, as well as the length of time until subjective improvement of the typical distressing symptoms were recorded in an unblinded fashion. We aimed to include only cases of adenoviral infection with a potential corneal involvement, avoiding cases of only mild adenoviral conjunctivitis. Therefore, we only included patients with marked conjunctival injection, swelling of the eyelids, and epiphora. Patients with any other ocular anterior segment morbidity, pregnant women, or patients using any other topical ocular medication were excluded from analysis. In all patients, diagnosis was confirmed by an in-office lateral flow immunoassay, RPS Adeno Detector (Rapid Pathogen Screening Inc., Sarasota, Florida, USA). The test is capable of detecting all 53 adenoviral serotypes by targeting the conserved hexon protein. Compared with PCR, the sensitivity was reported to be $89 \%$ and the specificity $94 \%{ }^{21}$ Patients were included in the statistical analysis if they were receiving either one of the 3 following treatment regimens, and were examined in the first and third weeks of therapy:

1. Topical 1\% prednisolone acetate+non-preserved artificial tears (Group 1)

2. Topical 2\% Cyclosporine A+non-preserved artificial tears (Group 2)

3. Only non-preserved artificial tears (Group 3)

The patients were not randomised for a particular treatment, because of the retrospective nature of the study. All patients were examined by one of the three experienced ophthalmologists (DDA, LA or EŞÖ). The choice of the treatment regime at the time was dependant on the clinical preference of the attending ophthalmologist and the preference of the patient. Topical Cyclosporine A treatment was preferred mostly by patients with a relatively higher socioeconomic status, due to the higher cost of the treatment. Topical Cyclosporine A $2 \%$ was compounded in a carboxymethylcellulose vehicle (noncommercialized; prepared in the pharmacy of Baskent University Hospital, Ankara, Turkey). The $2 \%$ dosage was preferred because it was the highest dosage reported to be used topically for epidemic keratoconjunctivitis ${ }^{19}$ without any systemic or local side effects, except for a case of childhood phlyctenular keratoconjunctivitis who developed a generalised skin rash under CsA $2 \%$ therapy. ${ }^{22}$ The eye with a worse clinical picture was included in bilateral cases.

A potent corticosteroid formulation was selected for the treatment, since short-term treatment of AAK with topical corticosteroids of limited potency may also delay viral clearance, similar to more potent formulations. ${ }^{23}$ The following parameters were recorded by a clinical score similar to described by Hillencamp at al. previously: ${ }^{24}$

1. Conjunctival injection: $0=$ no, $1=$ mild, $2=$ severe

2. Conjunctival chemosis: $0=$ no, $1=$ mild, $2=$ severe

3. Corneal subepithelial infiltrates: $0=$ no, $1=$ few $(\leqslant 10)$, $2=$ many $(>10)$

4. Schirmer's test (with topical anaesthesia): $0=>15 \mathrm{~mm}$, $1=5-15 \mathrm{~mm}, 2=<5 \mathrm{~mm}$

The sum of these clinical scores was calculated for each visit. 
All data were analysed for homogeneity of variance. The Kruskal-Wallis Test was used to evaluate the group differences for quantitative variables including age, sum of clinical scores, OSDI score, visual acuity, intraocular pressure, and the time until subjective improvement of the symptoms. The Mann-Whitney $U$ test was used to determine the specific inter-group differences when there was a significant group difference determined by the Kruskal-Wallis Test. For categorical variables including clinical scores for conjunctival injection, conjunctival chemosis, corneal subepithelial infiltrates, and the Schirmer's test, the inter-group differences were analysed by Chi-square test. We used the SPSS 15.0 software (SPSS Inc., Chicago, IL, USA).

\section{Results}

Hundred and ten eyes of 110 patients $(\mathrm{M} / \mathrm{F}=54 / 66)$ with a minimum follow-up duration of three weeeks were included in the study. All patients were under palliative therapy with non-preserved artificial tears. Forty six patients $(41.8 \%)$ were receiving topical corticosteroids (Group 1), 32 (29\%) were receiving topical 2\% Cyclosporin A (Group 2) and 32 (29\%) were using only artificial tears (Group 3). All patients received topical lubricants for $33.6 \pm 5.6$ days after the initial diagnosis. The mean duration of treatment with topical corticosteroids was $8.3 \pm 2.7$ days in Group 1, and the mean duration of treatment with topical CsA was $27.5 \pm 4.8$ days in Group 2. The mean age of the patients was (mean \pm SD) $35.2 \pm 15.8,33.4 \pm 14.6,31.9 \pm 16.5$ years in Groups 1, 2, and 3, respectively $(P=0.60)$.
At presentation, there was no significant difference among groups in terms of Snellen Best Corrected Visual Acuity, intraocular pressure (IOP), OSDI scores or the mean sum of clinical scores $(P=0.18 ; 0.55 ; 0.47$ and 0.06 respectively). There was no significant difference between the mean Snellen best corrected visual acuity of the 3 groups, at the first $(P=0.16)$ and third $(P=0.84)$ week of treatment. There was no decrease in visual acuity with respect to the initial examination in any patient, except for one patient in Group 1 who had a loss of one line due to the development of subepithelial infiltrates which were unresponsive to medical treatment. The mean and SD of the variables and the respective $p$ values for IOP, OSDI scores and sum of clinical scores at day 7 and 21, as well as the length of time until the resolution of the subjective symptoms in each group are given in Table 1.

There were significant inter-group differences for the OSDI scores at days $7(P=0.04)$ and $21(P=0.03)$. The mean sum of clinical scores were also significantly different between groups at day $21(P=0.02)$. There was also significant difference between groups in terms of the mean length of time until the resolution of the subjective symptoms $(P<0.01)$. The results of the analysis for the evaluation of inter-group differences for the variables with a significant group difference are given in Table 2.

The Ocular Surface Disease Index score at day 7 was the highest in Group $3(52.4 \pm 16.4)$ and the lowest in Group $1(41.0 \pm 21.3)(P=0.01)$. At day 21 , the OSDI score was also the highest in Group $3(32.3 \pm 13.0)$ and the lowest in Group $1(23.9 \pm 13.2)(P<0.01)$. At the 7 th and 21st days, Group 2 was not significantly different from Groups 1 and 3 in terms of OSDI scores.

Table 1 The mean and SD of the variables and the respective $p$ values for IOP (mmHg), OSDI scores and sum of clinical scores at day 7 and 21 in each group

\begin{tabular}{lcccc}
\hline & Group 1 (mean \pm SD) & Group 2 (mean \pm SD) & Group 3 (mean \pm SD) & P value \\
\hline IOP (mmHg) (Day 7) & $17.3 \pm 3.3$ & $16.2 \pm 3.5$ & $16.9 \pm 2.7$ & 0.247 \\
OSDI score (Day 7) & $41.0 \pm 21.3$ & $48.6 \pm 21.2$ & $52.4 \pm 16.4$ & $0.041^{\mathrm{a}}$ \\
Sum of CS (Day 7) & $2.0 \pm 1.3$ & $2.5 \pm 1.3$ & $3.0 \pm 2.1$ & 0.097 \\
IOP (mmHg) (Day 21) & $16.8 \pm 2.8$ & $15.6 \pm 3.0$ & $16.4 \pm 1.9$ & 0.175 \\
OSDI score (Day 21) & $23.9 \pm 13.2$ & $26.9 \pm 12.4$ & $32.3 \pm 13.0$ & $0.026^{\mathrm{a}}$ \\
Sum of CS (Day 21) & $0.7 \pm 0.8$ & $1.5 \pm 1.1$ & $1.9 \pm 1.2$ & $0.016^{\mathrm{a}}$ \\
Time until the resolution of the symptoms (Days) & $9.5 \pm 4.9$ & $11.2 \pm 4.9$ & $13.3 \pm 4.2$ & $0.005^{\mathrm{a}}$ \\
\hline
\end{tabular}

Abbreviations: CS, Clinical score; IOP, Intraocular pressure; OSDI, Ocular Surface Disease Index. aSignificant difference.

Table 2 The results of the analysis ( $p$ values) of specific inter-group differences for the variables with a significant group difference

\begin{tabular}{|c|c|c|c|c|}
\hline & OSDI score (Day 7) & OSDI score (Day 21) & Sum of CS (Day 21) & $\begin{array}{l}\text { Time until the resolution of the } \\
\text { subjective symptoms (Days) }\end{array}$ \\
\hline Group 1-2 $P$ values & 0.107 & 0.246 & 0.554 & 0.152 \\
\hline Group $1-3 P$ values & $0.010^{\mathrm{a}}$ & $0.009^{\mathrm{a}}$ & $0.011^{\mathrm{a}}$ & $0.001^{\mathrm{a}}$ \\
\hline Group 2-3 $P$ values & 0.479 & 0.076 & $0.028^{\mathrm{a}}$ & $0.050^{\mathrm{a}}$ \\
\hline
\end{tabular}

Abbreviations: CS, Clinical score; OSDI, Ocular Surface Disease Index. ${ }^{\text {a }}$ Significant difference. 
The mean sum of clinical scores was highest in Group 3 at days 7 and 21. At day 7 this difference was not significant $(P=0.10)$; however at day 21 there was a significant difference $(P=0.02)$. At day 21 , the mean sum of clinical scores in Group $3(1.9 \pm 1.2)$ was significantly higher than Groups $1(0.7 \pm 0.8)$ and $2(1.5 \pm 1.1)(P=0.01$ and 0.03 , respectively). There was no significant difference between the mean sum of clinical sores of Group 1 and Group 2 at any visit.

The mean time until the resolution of the subjective symptoms was significantly different between groups $(P=0.005)$. It was the lowest in Group 1 with $9.5 \pm 4.9$ days and highest in Group 3 with $13.3 \pm 4.2$ days $(P=0.001)$. There was no significant difference between Groups 1 and $2(P=0.152)$. The difference between Groups 2 and 3 was tenuous at $P=0.050$.

The clinical scores for conjunctival injection, conjunctival chemosis, corneal subepithelial infiltrates (CSE), and the Schirmer's test in 3 groups were also seperately analysed for each visit. At the initial visit, there was no significant difference between groups in terms of each clinical score. There was no significant difference among groups in terms of clinical scores for conjunctival injection, conjunctival chemosis, and Schirmer's test at any visit.

There was no significant difference between the clinical scores for CSE of the 3 groups on day $7(P=0.903)$ with the percentage of the patients with CSE being $46.4 \%$ in Group 1, 45.3\% in Group 2, and 51.3\% in Group 3. However, there was a statistically significant difference between the 3 groups at day $21(P=0.006)$. On that day, the percentage of the patients with CSE was the highest in Group 3 (63.4\%; $n: 20)$ and the lowest in Group 1 (42.5\%; $n: 14)$. The percentage of the patients with CSE in Group 2 (51.6\%; n: 16) was higher than Group 1 and lower than Group 3. Figure 1 shows the mean scores for CSE on the first, seventh and twenty first days in all groups. Charts of patients in Groups 1 and 2 with persistent CSE at the third week ( $n$ : 30$)$ were further examined for long term followup results. Eight patients were lost from follow-up. The remaining 22 patients were followed up for a minimum of 3 months (103.2 \pm 8.4 days). Persistent CSE were treated with low-potency topical corticosteroids in 12 patients and topical $2 \%$ CsA in 10 patients. Four patients from the group receiving topical corticosteroids and three patients from the group receiving topical $2 \% \mathrm{CsA}$ had persistent CSE at the end of the follow-up period.

None of the patients in the three groups experienced any local or systemic side effects necessitating the cessation of therapy or a change in the treatment regimen, except for two patients in Group 1 who had increased intraocular pressure at the first week visit. Intraocular pressure was normalised in these patients upon cessation of topical corticosteroids.

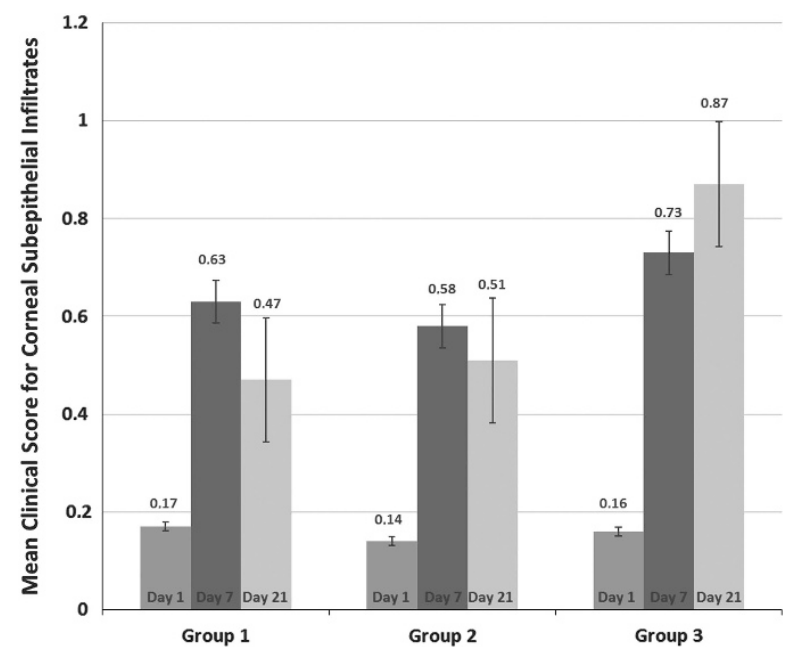

Figure 1 The mean clinical scores for corneal subepithelial infiltrates on the first, seventh and twenty first days in Groups 1, 2 and 3. (Error bars indicating standard error of mean.)

\section{Discussion}

To the best of our knowledge, this is the first study evaluating the performance of topical 2\% Cyclosporin A in the treatment of acute adenoviral conjunctivitis. We also compared the clinical outcomes of patients receiving topical corticosteroids, Cyclosporin A and only palliative treatment. We observed that the symptoms were less severe and had a shorter duration with topical corticosteroids and CsA when compared with palliative therapy and topical 2\% CsA had an inhibitory effect on the development of corneal subepithelial infiltrates when used in the acute phase of infection, similar to corticosteroids.

There is no currently available treatment that is effective against adenoviral keratoconjunctivitis. Despite the fact that this is a self-limiting disease, most affected individuals seek and receive treatment as a result of the severity of their symptoms. ${ }^{25}$ This may be the reason that $36 \%$ of eye care practitioners still prescribe topical corticosteroids for epidemic keratokonjunctivitis. ${ }^{26}$ In a randomised placebo-controlled trial including 111 viral conjunctivitis patients, the group receiving topical dexamethasone was significantly more likely to report that they felt the drops helped than the group receiving vehicle control drops. ${ }^{4}$ However, three other older reports comparing topical steroids versus topical lubricants found no difference in the amount of subepithelial infiltrates and punctate epithelial keratitis, course of the disease and symptoms. ${ }^{14,27,28}$

Cyclosporine is another agent explored for treating symptoms caused by AAK. One study found accelerated subjective improvement of local symptoms with $1 \%$ cyclosporine eyedrops (4 times a day for 21 days), as 
compared with $0.2 \%$ cidofovir or a combination of $0.2 \%$ cidofovir $+1 \%$ cyclosporine. ${ }^{20}$ In our study, we observed that patients receiving topical corticosteroids experienced the symptoms for a shorter period of time when compared with the palliative treatment group. The mean time until the resolution of the subjective symptoms was significantly higher in patients receiving only artificial tears and palliative treatment $(13.3 \pm 4.2$ days $)$, when compared with patients receiving topical $2 \%$

Cyclosporine A (11.2 \pm 4.9 days) and topical 1\% prednisolone acetate $(9.5 \pm 4.9$ days). Patients receiving topical Cyclosporine A had a shorter duration of symptoms when compared with patients receiving palliative therapy as well.

We used the OSDI questionnaire in an attempt to evaluate the symptomatic differences between groups during the treatment period. The mean OSDI scores were lower in patients receiving topical corticosteroids at every visit and this difference was significant at days 7 and 21 . In patients receiving palliative treatment, the OSDI scores were significantly higher than the patients receiving topical corticosteroids at both visits. Patients receiving topical CsA had similar OSDI scores to patients receiving palliative treatment at each visit. The relatively high OSDI scores in patients receiving topical CsA might be related to the slight ocular discomfort caused by the cyclosporine eye drops which was also reported by some patients in this group.

The mean sum of clinical scores was highest in patients receiving palliative treatment at all control visits and this differrence was significant at day 21 . There was no significant difference between the mean sum of clinical scores of patients receiving topical corticosteroids and topical CsA at any visit. When we analysed each clinical score seperately, there was no significant difference among groups in terms of clinical scores for conjunctival injection, conjunctival chemosis, and Schirmer's test at any visit. This is similar to the findings of the previous literature comparing topical corticosterois with lubricants. ${ }^{27}$

Corticosteroids are advised to be reserved until patients present with a pseudomembrane or symptomatic subepithelial infiltrates, however it is not known if they have a preventive effect on the development of corneal SEI when used in the acute phase of infection. ${ }^{29}$ Cyclosporine was specifically explored for treating symptoms caused by subepithelial infiltrates. ${ }^{12,13,19,20}$ One percent CsA administered 4 times a day for 21 days during acute infection produced earlier subjective improvement of local symptoms and a trend toward a lower incidence of corneal opacities. ${ }^{20}$ In a second study, the majority of patients with longstanding established CSE were successfully treated with $2 \%$ CsA with reduction or elimination of SEIs and successful weaning off therapy. ${ }^{19}$ Similarly, Levinger et al ${ }^{13}$ reported improvement in vision and satisfaction with topical $1 \%$ CSA in 9 patients with subepithelial corneal infiltrates secondary to adenoviral keratoconjunctivitis.

Romanowski et al found that treatment with both 2.0 and $0.5 \%$ CsA in the rabbit model significantly reduced the formation of SEIs. ${ }^{12}$ In our study, at day 21, the percentage of the patients with CSE was highest in the palliative treatment group (63.4\%) and lowest in topical corticosteroid group (42.5\%). The percentage of the patients with CSE in the topical Cyclosporine group (51.6\% at day 21) was higher than patients receiving topical corticosteroids and lower than patients receiving topical lubricants. As a result it appears that topical corticosteroids have an inhibitory effect on the development of CSE when used in the acute phase of infection and topical Cyclosporine A has a similar inhibitory effect as well, although less potent.

There are two potential side effects from using topical steroids to treat viral conjunctivitis: the promotion of viral replication and the associated exacerbation of unrecognised HSV infection. In our study, the diagnosis was confirmed by an in-office lateral flow immunoassay, RPS Adeno Detector (Rapid Pathogen Screening Inc., Sarasota, Florida, USA) in all patients thus, other causes of viral conjunctivitis were ruled out. It is known that topical steroids increase and prolong viral shedding in rabbit models of adenoviral conjunctivitis, even when given for as little as 3 days. ${ }^{30}$ Thus steroids could exacerbate adenovirus infection in humans and cause epidemic outbreaks. The same effect has been shown with topical Cyclosporine A. ${ }^{12}$ Patients presenting with AAK should be informed about the highly contagious nature of the disease and the necessary precautions that should be taken to minimise the spread of the disease in an attempt to prevent epidemic outbreaks, especially in cases treated with topical corticosteroids or CsA. The risk of using corticosteroid or cyclosporine eye drops and possibly prolonging and facilitating the spread of the infection must be weighed against the clinical benefit of symptomatic relief and the inhibitory effect on corneal complications.

As a result, the symptoms were observed to be less severe and had a shorter duration in patients receiving topical corticosterois and topical CsA when compared with patients receiving only palliative treatment. Furthermore, it appears that topical 2\% CsA has an inhibitory effect on the development of CSE when used in the early phase of infection, similar to topical corticosteroids. This is a complication causing significant potential of morbidity and visual loss. Topical $2 \%$ Cyclosporin A had a similar effect to corticosteroids, with the advantage of being a steroid-sparing agent. The most important disadvantage of CsA therapy is the high cost 
when compared with topical corticosteroids and lubricants. Further studies should focus on the use of topical CsA for acute adenoviral keratoconjunctivitis and prevention of its corneal complications, in different doses and frequencies, as well as comparative studies with corticosteroids including their effect on viral shedding.

\section{Summary}

\section{What was known before}

- The use of topical steroids is recommended only to reduce scarring in severe cases of acute adenoviral keratoconjunctivitis (AAK) with marked chemosis, lid swelling, epithelial sloughing, or membranous conjunctivitis. However, short term topical steroids are still frequently used with an aim to decrease patient discomfort.

- Topical Cyclosporin A, in different concentrations ( 1 and $2 \%$ ), is effective in the treatment of subepithelial infiltrates during the chronic phase of AAK.

\section{What this study adds}

- The symptoms of acute adenoviral keratoconjunctivitis AAK) may be less severe and have a shorter duration in patients receiving topical corticosterois and topical CsA when compared with patients receiving only topical lubricants.

- Clinical signs associated with corneal subepthelial infiltrates may be less severe in patients receiving topical corticosteroids, as well as topical 2\% Cyclosporin A.

\section{Conflict of interest}

The authors declare no conflict of interest.

\section{Acknowledgements}

There are no sources of support or funding for this study, including sponsorship (eg, university, charity, commercial organisation). Topical Cyclosporine A 2\% was compounded in a carboxymethylcellulose vehicle, prepared in the pharmacy of Baskent University Hospital, Ankara, Turkey.

\section{References}

1 Fuchs E. Keratitis punctata superficialis. Wien Klin Wochenschr 1889; 44: 837-842.

2 Dawson CR, Sheppard J. Follicular conjunctivitis. In: Tasman W, Jaeger EA (eds). Duane's Clinical Ophthalmology, Vol. 4, chap. 7. Lippincott-Raven: Philadelphia, 1995, pp 2-8.

3 American Academy of Ophthalmology. Preferred Practice Pattern, Adenoviral Conjunctivitis. Available at: http:/ /one. aao.org/preferred-practice-pattern/conjunctivitis-ppp-2013. (Accessed: 12.10.2014).
4 Wilkins MR, Khan S, Bunce C, Khawaja A, Siriwardena D, Larkin DF. A randomised placebo-controlled trial of topical steroid in presumed viral conjunctivitis. Br J Ophthalmol 2011; 95: 1299-1303.

5 Calne RY, White DJ, Thiru S, Evans DB, McMaster P, Dunn DC et al. Cyclosporin A in patients receiving renal allografts from cadaver donors. Lancet 1978; 2: 1323-1327.

6 Schultz C. Safety and efficacy of cyclosporine in the treatment of chronic dry eye. Ophthalmol Eye Dis 2014; 6: $37-42$.

7 Erdinest N, Solomon A. Topical immunomodulators in the management of allergic eye diseases. Curr Opin Allergy Clin Immunol 2014; 14: 457-463.

8 Liegner JT, Yee RW, Wild JH. Topical cyclosporine therapy for ulcerative keratitis associated with rheumatoid arthritis. Am J Ophthalmol 1990; 109: 610-612.

9 Reinhard T, Sundmacher R. Topical cyclosporin A in Thygeson's superficial punctate keratitis. Graefes Arch Clin Exp Ophthalmol 1999; 237: 109-112.

10 Okumus S, Coskun E, Tatar MG, Kaydu E, Yayuspayi R, Comez A et al. Cyclosporine a $0.05 \%$ eye drops for the treatment of subepithelial infiltrates after epidemic keratoconjunctivitis. BMC Ophthalmol 2012; 12: 42.

11 Jeng BH, Holsclaw DS., Cyclosporine A. 1\% eye drops for the treatment of subepithelial infiltrates after adenoviral keratoconjunctivitis. Cornea 2011; 30: 958-961.

12 Romanowski EG, Pless P, Yates KA, Gordon YJ. Topical cyclosporine A inhibits subepithelial immune infiltrates but also promotes viral shedding in experimental adenovirus models. Cornea 2005; 24: 86-91.

13 Levinger E, Slomovic A, Sansanayudh W, Bahar I, Slomovic AR. Topical treatment with $1 \%$ cyclosporine for subepithelial infiltrates secondary to adenoviral keratoconjunctivitis. Cornea 2010; 29: 638-640.

14 Ward JB, Siojo LG, Waller SG. A prospective, masked clinical trial of trifluridine, dexamethasone, and artificial tears in the treatment of epidemic keratoconjunctivitis. Cornea 1993; 12: 216-221.

15 Little JM, Lorenzetti DW, Brown DC, Schweem HH, Jones BR, Kaufman HE. Studies of adenovirus type 3 infection treated with methisazone and trifluorothymidine. Proc Soc Exp Biol Med 1968; 127: 1028-1032.

16 Waring G. Use of vidarabine in epidemic keratoconjunctivitis due to adenovirus types $3,7,8$, and 19 . Am J Ophthalmol 1976; 82: 781-785.

17 Trinavarat A, Atchaneeyasakul L. Treatment of epidemic keratoconjunctivitis with $2 \%$ povidone-iodine: a pilot study. J Ocul Pharmacol Ther 2012; 28: 53-58.

18 Clement C, Capriotti JA, Kumar M, Hobden JA, Foster TP, Bhattacharjee PS et al. Clinical and antiviral efficacy of an ophthalmic formulation of dexamethasone povidone-iodine in a rabbit model of adenoviral keratokonjonctivitis. Invest Ophthalmol Vis Sci 2011; 52: 339-344.

19 Reinhard T, Godehardt E, Pfahl HG, Sundmacher R. Local cyclosporin A in nummuli after keratoconjunctivitis epidemica. A pilot study. Ophthalmologe 2000; 97: 764-768.

20 Hillenkamp J, Reinhard T, Ross RS, Böhringer D, Cartsburg $\mathrm{O}$, Roggendorf $\mathrm{M}$ et al. Topical treatment of acute adenoviral keratoconjunctivitis with $0.2 \%$ cidofovir and $1 \%$ cyclosporine: a controlled clinical pilot study. Arch Ophthalmol 2001; 119: 1487-1491.

21 Sambursky R, Tauber S, Schirra F, Kozich K, Davidson R, Cohen EJ. The RPS Adeno Detector for diagnosing 
adenoviral conjunctivitis. Ophthalmology 2006; 113: 1758-1764.

22 Doan S, Gabison E, Gatinel D, Duong MH, Abitbol O, Hoang-Xuan T. Topical cyclosporine A in severe steroiddependent childhood phlyctenular keratoconjunctivitis. Am J Ophthalmol 2006; 141: 62-66.

23 Romanowski EG, Yates KA, Gordon YJ. Topical corticosteroids of limited potency promote adenovirus replication in the Ad5/NZW rabbit ocular model. Cornea 2002; 21: 289-291.

24 Hillenkamp J, Reinhard T, Ross RS, Böhringer D, Cartsburg O, Roggendorf $\mathrm{M}$ et al. The effects of cidofovir $1 \%$ with and without cyclosporin a $1 \%$ as a topical treatment of acute adenoviral keratoconjunctivitis: a controlled clinical pilot study. Ophthalmology 2002; 109: 845-850.

25 Lenaerts L, Naesens L. Antiviral therapy for adenovirus infections. Antiviral Res 2006; 72: 172-180.
26 Kowalski RP, Foulks GN, Gordon YJ. An overview comparing treatment regimens for ocular infections: community versus academia. Ann Ophthalmol 2000; 32: 295-300.

27 Trauzettel-Klosinski S, Sundmacher R, Wigand R. The effects of topical steroids in epidemic kerato-conjunctivitis. Klin Monbl Augenheilkd 1980; 176: 899-906.

28 Laibson PR, Dhiri S, Oconer J, Ortolan G. Corneal infiltrates in epidemic keratoconjunctivitis. Response to double-blind corticosteroid therapy. Arch Ophthalmol 1970; 84: $36-40$.

29 Butt AL, Chodosh J. Adenoviral keratoconjunctivitis in a tertiary care eye clinic. Cornea 2006; 25: 199-202.

30 Romanowski EG, Yates KA, Gordon YJ. Short-term treatment With a potent topical corticosteroid of an acute ocular adenoviral infection in the New Zealand white rabbit. Cornea 2001; 20: 657-660. 